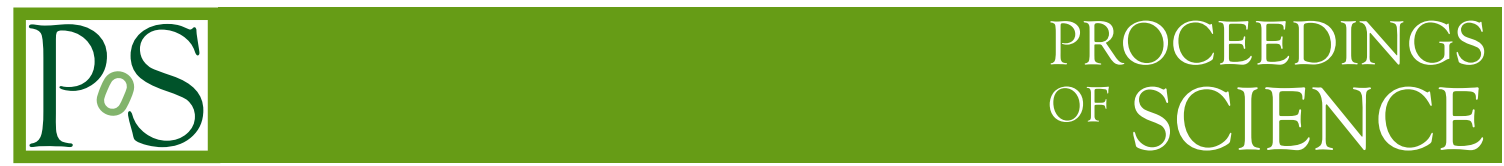

\title{
NSPT calculations in the Schrödinger Functional formalism
}

\section{Torrero*, G.S. Bali}

Institute for Theoretical Physics, University of Regensburg, 93040, Regensburg, Germany

E-mail: christian.torrerodphysik.uni-regensburg.de

gunnar.baliaphysik. uni-regensburg.de

Within the framework of the Schrödinger Functional (SF), we outline how to combine Numerical Stochastic Perturbation Theory (NSPT) and PCAC relations to determine the two-loop contributions to the improvement coefficients $c_{A}$ and $c_{S W}$ for Sheikholeslami-Wohlert-Wilson fermions.

The XXVII International Symposium on Lattice Field Theory - LAT2009

July 26-31 2009

Peking University, Beijing, China

\footnotetext{
* Speaker.
} 


\section{Introduction}

As it is well-known, in the improvement approach à la Symanzik [1] the lattice QCD action has to be provided with an extra irrelevant contribution, the so-called Sheikholeslami-Wohlert term [2]. In Perturbation Theory (PT), it features a scalar coefficient $c_{S W}$ which can be Taylor-expanded in powers of the bare coupling $g_{0}$ as

$$
c_{S W}=c_{S W}^{(0)}+c_{S W}^{(1)} g_{0}^{2}+c_{S W}^{(2)} g_{0}^{4}+\mathscr{O}\left(g_{0}^{6}\right) .
$$

The zero- and one-loop coefficients have already been determined for different lattice actions [3][4] while $c_{S W}^{(2)}$ is still unknown: the final aim of this project is precisely to estimate it by combining the Schrödinger Functional formalism (SF) and the PCAC relations in the same spirit as [5] and [6] where $c_{S W}^{(0)}$ and $c_{S W}^{(1)}$ were successfully recovered.

The main difference with these two latter seminal papers lies in the fact that observables are evaluated perturbatively without following a diagrammatic approach but rather by means of Numerical Stochastic Perturbation Theory (NSPT), a computer algorithm characterized by a Langevinlike evolution of the system.

\section{Theoretical aspects - part I (basics)}

The lattice formulation of QCD we adopt is that of Wilson: a concrete expression of the wellknown contributions to the action - namely the gauge $\left(S_{G}\right)$, fermionic $\left(S_{F}\right)$ and SheikholeslamiWohlert $\left(S_{S W}\right)$ term - can be found in [5] whose notations and conventions inspire nearly all the formulae appearing in this and the next section ${ }^{1}$.

A suitable observable to study in order to evaluate $c_{S W}^{(2)}$ is provided by the quark mass $m_{q}$ which can be conveniently computed by means of the lattice PCAC relation reading, ${ }^{2}$

$$
\frac{1}{2}\left(\partial_{0}^{R}+\partial_{0}^{L}\right)\left\langle A_{0}^{b}(n) \mathbb{O}\right\rangle=2 m_{q}\left\langle P^{b}(n) \mathbb{O}\right\rangle,
$$

where $\mathbb{O}$ is any product of fields located at nonzero distance from $n, \partial_{0}^{R}\left(\partial_{0}^{L}\right)$ is the lattice right (left) derivative in the time direction and

$$
A_{0}^{b}(n)=\sum_{f, g}^{N_{f}} \bar{\psi}^{f}(n) \gamma_{\mu} \gamma_{5} \frac{1}{2} \tau_{f g}^{b} \psi^{g}(n), \quad P^{b}(n)=\sum_{f, g}^{N_{f}} \bar{\psi}^{f}(n) \gamma_{5} \frac{1}{2} \tau_{f g}^{b} \psi^{g}(n),
$$

where $\tau^{b}$ is a matrix acting on flavour degrees of freedom ${ }^{3}$.

In order to fix $c_{S W}^{(2)}$, one requires $m_{q}$ to be independent of contributions of order $a$ : however, to achieve full improvement Eq.(2.1) has to be modified to,

$$
\frac{1}{2}\left(\partial_{0}^{R}+\partial_{0}^{L}\right)\left\langle A_{0}^{b}(n) \mathbb{O}\right\rangle+c_{A} \partial_{0}^{L} \partial_{0}^{R}\left\langle P^{b}(n) \mathbb{O}\right\rangle=2 m_{q}\left\langle P^{b}(n) \mathbb{O}\right\rangle,
$$

\footnotetext{
${ }^{1}$ More generally, we stick to the setup outlined in sections 2, 4 and 6 of [5].

${ }^{2}$ From now on, the time direction will be assigned the subscript 0 .

${ }^{3}$ Spin and colour subscripts will be usually left implicit in order to ease the notation.
} 
where $c_{a}$ is a second improvement coefficient which, just like $c_{S W}$, can also be decomposed as $c_{A}=c_{A}^{(0)}+c_{A}^{(1)} g_{0}^{2}+c_{A}^{(2)} g_{0}^{4}+\mathscr{O}\left(g_{0}^{6}\right)$. Once again, the first unknown contribution is at two-loop level: see [5] and [6] for the determination of $c_{A}^{(0)}$ and $c_{A}^{(1)}$.

The second main theoretical ingredient of the present strategy is given by the Schrödinger Functional: assuming the time coordinate ranges from 0 to $T$ and labelling the space coordinates as $\vec{n}$, it consists of replacing the usual periodic boundaries by Dirichlet conditions along the time direction, namely,

$$
\left.U_{k}(n)\right|_{n_{0}=0} \rightarrow W_{k}(\vec{n}),\left.\quad U_{k}(n)\right|_{n_{0}=T} \rightarrow W_{k}^{\prime}(\vec{n}) \quad(k=1,2,3),
$$

for the gauge degrees of freedom ${ }^{4}$ and $\left(P_{ \pm}=\left(\mathbb{I} \pm \gamma_{0}\right) / 2\right.$ with $\mathbb{I}$ being the identity matrix $)$

$$
\begin{array}{rlrl}
\left.\psi^{f}(n)\right|_{n_{0}=0} & \rightarrow \rho^{f}(\vec{n})=\left.P_{+} \psi^{f}(n)\right|_{n_{0}=0}, & \left.\psi^{f}(n)\right|_{n_{0}=T} \rightarrow \rho^{\prime f}(\vec{n})=\left.P_{+} \psi^{f}(n)\right|_{n_{0}=T}, \\
\left.\bar{\psi}^{f}(n)\right|_{n_{0}=0} \rightarrow \bar{\rho}^{f}(\vec{n})=\left.P_{+} \bar{\psi}^{f}(n)\right|_{n_{0}=0}, & \left.\bar{\psi}^{f}(n)\right|_{n_{0}=T} \rightarrow \bar{\rho}^{\prime f}(\vec{n})=\left.P_{+} \bar{\psi}^{f}(n)\right|_{n_{0}=T},
\end{array}
$$

for fermions: boundary fields $W, W^{\prime}, \rho, \bar{\rho}, \rho^{\prime}$ and $\bar{\rho}^{\prime}$ will be defined later on.

Due to the Schrödinger Functional formalism, the three contributions to the lattice QCD action get modified as follows:

- the gauge part $S_{G}$ becomes

$$
S_{G}=\beta \sum_{\substack{n, \mu, v \\ \mu>v}} \omega_{\mu v}(n)\left(1-\frac{T r}{2 N_{c}}\left[U_{\mu v}(n)+U_{\mu v}^{\dagger}(n)\right]\right),
$$

where the weight $\omega_{\mu v}(n)$ for the lattice plaquette $U_{\mu v}(n)$ is 1 everywhere except for the spatial plaquette at $n_{0}=0$ and $n_{0}=T$ whose $\omega_{\mu v}(n)$ reads $\frac{1}{2}$;

- the fermionic part $S_{F}$ remains in principle unchanged; anyway, in order to have one more parameter to play with, an additional phase $e^{i \theta_{\mu} / L_{\mu}}$ is introduced in the definition of the lattice covariant derivatives within the Wilson-Dirac operator: in practice, gauge fields $U_{\mu}(n)$ appearing in $S_{F}$ are replaced by,

$$
U_{\mu}(n) \rightarrow e^{i \theta_{\mu} / L_{\mu}} U_{\mu}(n),
$$

with $\theta_{0}=0$ and $-\pi<\theta_{k} \leq \pi$ for $k=1,2,3$;

- the clover term is set to 0 for all those lattice points with $n_{0}=0$ or $n_{0}=T$.

\footnotetext{
${ }^{4}$ Gauge fields along the time direction, defined for $0 \leq n_{0}<T$, have no constraints on them. It turns out that $W$ and $W^{\prime}$ can sloppily be written as $W=\mathscr{P} e^{\int C}$ and $W=\mathscr{P} e^{\int C^{\prime}}-$ see section 6 of [5] for notations and a more careful and detailed treatment of this topic - where $C$ and $C^{\prime}$ play a similar role as the background field in classical physics: in what follows we will refer to the case $C=C^{\prime}=0$ as the trivial background.
} 


\section{Theoretical aspects - part II (details)}

Before outlining the procedure that should lead to an estimate of $c_{S W}^{(2)}$, let us give a precise shape to the observable $\mathbb{O}$ appearing in Eq.(2.3): a convenient choice reads,

$$
\mathbb{O}=a^{6} \sum_{f, g}^{N_{f}} \sum_{\vec{m}, \vec{m}^{\prime}} \bar{\varsigma}^{f}(\vec{m}) \gamma_{5} \frac{1}{2} \tau_{f g}^{b} \varsigma^{g}\left(\vec{m}^{\prime}\right)
$$

where

$$
\varsigma^{f}(\vec{m})=\frac{\delta}{\delta \bar{\rho}^{f}(\vec{m})}, \quad \bar{\varsigma}^{f}(\vec{m})=-\frac{\delta}{\delta \rho^{f(\vec{m})}}
$$

After first plugging Eq.(3.1) into Eq.(2.3), then letting the derivatives with respect to $\rho$ and $\bar{\rho}$ act on the Boltzmann factor and finally setting all the fermionic boundary fields to zero, some algebra allows one to write

$$
m_{q}=\frac{\frac{1}{2}\left[\frac{1}{2}\left(\partial_{0}^{R}+\partial_{0}^{L}\right) f_{A}+c_{A} \partial_{0}^{L} \partial_{0}^{R} f_{P}\right]}{f_{P}}
$$

with $^{5}$

$$
\begin{aligned}
f_{A} & =\frac{1}{12} \sum_{\vec{m}, \vec{m}^{\prime}}\left\langle H_{[(\vec{m}+\hat{0}) \omega c, n \varepsilon e]}^{l f}\left(\gamma_{0}\right)_{\varepsilon \beta} \tau_{f g}^{b}\left(P_{-}\right)_{\omega \sigma} J_{\left[\left(\vec{m}^{\prime}+\hat{0}\right) \sigma c, n \beta e\right]}^{g h} \tau_{h l}^{b}\right\rangle_{G}, \\
f_{P} & =\frac{1}{12} \sum_{\vec{m}, \vec{m}^{\prime}}\left\langle H_{[(\vec{m}+\hat{0}) \omega c, n \varepsilon e]}^{l f} \tau_{f g}^{b}\left(P_{-}\right)_{\omega \sigma} J_{\left[\left(\vec{m}^{\prime}+\hat{0}\right) \sigma c, n \varepsilon e\right]}^{g h} \tau_{h l}^{b}\right\rangle_{G},
\end{aligned}
$$

with

$$
\begin{aligned}
& H_{[(\vec{m}+\hat{0}) \omega c, n \varepsilon e]}^{l f}=\left[U_{0}(\vec{m})\right]_{c b}\left(\widetilde{M}^{-1}\right)_{[(\vec{m}+\hat{0}) \omega b, n \varepsilon e]}^{l f}, \\
& J_{\left[\left(\vec{m}^{\prime}+\hat{0}\right) \sigma c, n \beta e\right]}^{g h}=\left[U_{0}\left(\vec{m}^{\prime}\right)\right]_{c d}^{*}\left(\widetilde{M}^{-1^{*}}\right)_{\left[\left(\vec{m}^{\prime}+\hat{0}\right) \sigma d, n \beta e\right]}^{g h},
\end{aligned}
$$

where $\widetilde{M}$ is the overall fermionic opearator in the lattice action.

$f_{A}, f_{P}$ and $m_{q}$ depend on the lattice spacing $a$, the lattice extents $L_{\mu}$, the bare coupling $g_{0}$, the gauge fields $W$ and $W^{\prime}$, the angles $\theta_{k}$ (from now on, we will set the latter equal to a common value $\theta$ ) and the improvement coefficients: recalling that the approach is perturbative, we can write ${ }^{6}$,

$$
m_{q}\left(L, \theta, x_{0}, g_{0}, a\right)=m_{q}^{(0)}\left(L, \theta, x_{0}, a\right)+m_{q}^{(2)}\left(L, \theta, x_{0}, a\right) g_{0}^{2}+m_{q}^{(4)}\left(L, \theta, x_{0}, a\right) g_{0}^{4}+\mathscr{O}\left(g_{0}^{6}\right),
$$

\footnotetext{
${ }^{5}$ The subscript " $G$ " stands for the mean over gauge degrees of freedom. Here and in Eqs.(3.6)-(3.7) repeated indices are summed over. Moreover, from now on we tacitly assume that all quantities are rescaled with $a$ to be dimensionless.

${ }^{6}$ We make the dependence on $W, W^{\prime}, c_{S W}$ and $c_{A}$ implicit not to overwhelm the notation; at the same time, we drop the subscript on the lattice extents for a reason that will become clear soon.
} 
and in turn, thanks to dimensional analysis

$$
m_{q}^{(k)}\left(L, \theta, x_{0}, a\right)=d_{L}\left(c_{S W}^{(i \leq k)}, c_{A}^{(i \leq k)}\right) \frac{a}{L}+d_{x_{0}}\left(c_{S W}^{(i \leq k)}, c_{A}^{(i \leq k)}\right) \frac{a}{x_{0}}+d_{\theta}\left(c_{S W}^{(i \leq k)}, c_{A}^{(i \leq k)}\right) \frac{a \theta}{L}+\mathscr{O}\left(a^{2}\right)
$$

This formula can actually be simplified by setting the $L_{k}$ 's to the same value $L$, putting $L_{0}=2 L$ and choosing $n_{0}=L$ : thus, the corrections in $a$ to $m_{q}^{(k)}$ will be grouped together into a single one proportional to $a / L$. Since the aim of improvement is to get rid of lattice artifacts of order $a$, it is reasonable to estimate $c_{S W}^{(2)}$ by requiring the only coefficient $d\left(c_{S W}^{(i \leq k)}, c_{A}^{(i \leq k)}\right)$ left in the formula above - after its reduction - to vanish. This can be achieved by the following steps: 1) fix $c_{S W}^{(2)}$ and $c_{A}^{(2)}$ arbitrarily after setting $c_{S W}^{(0)}, c_{S W}^{(1)}, c_{A}^{(0)}$ and $c_{A}^{(1)}$ to their known values; 2) perform simulations for different lattice extents keeping $\theta, W$ and $W^{\prime}$ constant; 3) fit the coefficient $\left.d\left(c_{S W}^{(2)}, c_{A}^{(2)}\right) ; 4\right)$ repeat the previous steps for different choices of $c_{S W}^{(2)}$ and $\left.c_{A}^{(2)} ; 5\right)$ collect the various estimates of $d\left(c_{S W}^{(2)}, c_{A}^{(2)}\right)$ and interpolate the values of $c_{S W}^{(2)}$ and $c_{A}^{(2)}$ for which $d\left(c_{S W}^{(2)}, c_{A}^{(2)}\right)$ vanishes.

Before ending this section, some remarks are in order.

The first term on the r.h.s.of Eq.(3.8) should normally correspond to the bare mass $\widehat{M}_{0}$ appearing in $S_{F}$; however, in the present setup, this is the case only if $\theta=0$ : we chose to set $\widehat{M}_{0}=0$ but to work with non-vanishing $\theta$ to avoid any infrared divergence.

Second, in Eq.(3.9) it is understood that mass counterterms - depending on $c_{S W}$ [7] - are subtracted. Otherwise $m_{q}^{(k)}$ would not be 0 in the large $L$ limit: this subtraction prevents extra improvement coefficients to appear (see section 3 in [5]) but, in practice, this should really matter only when working with renormalized quantities (while we deal with their bare counterparts).

Finally, it is possible to disentangle the effects of $c_{S W}^{(2)}$ and $c_{A}^{(2)}$ by means of $W$ and $W^{\prime}$ : in particular it turns out that, if the trivial background (see footnote 4 ) is set, only $c_{A}^{(2)}$ has an effect at two-loop level. We start with this choice of the boundary gauge fields to fix this coefficient, afterwards $W$ and $W^{\prime}$ will be changed to determine $c_{S W}^{(2)}$ thanks also to the by-then-known estimate of $c_{A}^{(2)}$.

\section{Numerical aspects}

Two more issues have still to be addressed about the present strategy, namely how configurations are generated and how the Wilson-Dirac operator is inverted to compute $f_{A}$ and $f_{P}$ eventually: to answer both, we must introduce some basics of $\mathrm{NSPT}^{7}$.

Its core is given by the Langevin evolution equation that, for lattice gauge variables ${ }^{8}$, reads

$$
\frac{\partial}{\partial t} U_{\mu}(n, t)=-i \sum_{A} T^{A}\left[\nabla_{n, \mu, A} S[U]+\eta_{\mu}^{A}(n, t)\right] U_{\mu}(n, t)
$$

where $t$ is an extra degree of freedom (which can be thought as a stochastic time), $S$ is the part of the lattice action depending on the $U$ 's, $\eta$ is a Gaussian noise while $\nabla$ stands for the group derivative

\footnotetext{
${ }^{7}$ See [8] and references therein for more details on this section in general.

${ }^{8}$ As usual, fermion fields are integrated out so that only gauge degrees of freedom have to be eventually treated.
} 
defined as (index " $A$ " is summed over),

$$
\mathscr{F}\left[e^{i \alpha^{A} T^{A}} U_{\mu}(n), U^{\prime}\right]=\mathscr{F}\left[U_{\mu}(n), U^{\prime}\right]+\alpha^{A} \nabla_{n, \mu, A} \mathscr{F}\left[U_{\mu}(n), U^{\prime}\right]+\ldots
$$

where $T^{A}$ are the generators of the algebra and $\mathscr{F}$ is a generic scalar function of both the variable $U_{\mu}(n)$ and some more labelled $U^{\prime}$ for short.

Given this setup, it can be shown that

$$
Z^{-1} \int[D U] O[U] e^{-S[U]}=\lim _{t \rightarrow \infty} \frac{1}{t} \int_{0}^{t} \mathrm{~d} t^{\prime}\left\langle O\left[U_{\eta}\left(t^{\prime}\right)\right]\right\rangle_{\eta}
$$

where $Z$ is the partition function and $O[U]$ a generic observable depending on the gauge fields.

Perturbation theory enters into play by formally expanding each gauge degree of freedom in powers of $\beta_{0}^{-1}$ - defined as $\beta_{0}=2 N_{c} / g_{0}^{2}$ being $N_{c}$ the number of colours - up to a given order $s$ as

$$
U_{\mu}(n, t)=\mathbb{I}+\sum_{k=1}^{s} \beta_{0}^{-\frac{k}{2}} U_{\mu}^{(k)}(n, t),
$$

and then plugging this Taylor series ${ }^{9}$ into Eq.(4.1): this results in a consistent hierarchical system of differential equations which can be numerically integrated by discretizing the stochastic time as $t=n \tau$ with $n$ integer. In practice, the system starts from an arbitrary configuration and evolves by means of the solution of the discretized counterpart of Eq.(4.1): the desired observable is then obtained by averaging its measurements on its plateau - recall the limit in $t$ in Eq.(4.3) ${ }^{10}$.

As for the inverse of the fermionic operator, the entries needed to get $f_{A}$ and $f_{P}$ can be computed by means of the following perturbative formulae

$$
\begin{aligned}
\widetilde{M}^{-1^{(0)}}= & \widetilde{M}^{(0)^{-1}}, \\
\widetilde{M}^{-1^{(1)}}= & -\widetilde{M}^{(0)^{-1}} \widetilde{M}^{(1)} \widetilde{M}^{(0)^{-1}}, \\
\widetilde{M}^{-1^{(2)}}= & -\widetilde{M}^{(0)^{-1}} \widetilde{M}^{(2)} \widetilde{M}^{(0)^{-1}}+ \\
& -\widetilde{M}^{(0)^{-1}} \widetilde{M}^{(1)} \widetilde{M}^{-1^{(1)}},
\end{aligned}
$$

where only the zeroth order of $\widetilde{M}$ has to be truly inverted: its expression for trivial $W$ and $W^{\prime}$ can be found in section 3.1 of [6].

\section{Preliminary results}

To test the correctness of the overall setup, we computed the one-loop contribution to $m_{q}$ without any counterterm subtraction for different choices of $\theta$ and $c_{S W}^{(0)}{ }^{11}$ and compared the results

\footnotetext{
${ }^{9}$ Strictly speaking, Eq.(4.3) is valid only if the boundary gauge fields are set to the identity as in this first part of the study; once that a non-trivial background field is introduced, the expansion would read $U_{\mu}(n, t)=\exp \left[\left(C_{k}^{\prime}-C_{k}\right) / T\right]$. $\cdot\left[\mathbb{I}+\sum_{k} \beta^{-\frac{k}{2}} U_{\mu}^{(k)}(n, t)\right]$ - consult section 6.2 in [5] for the meaning of the first term in this product.

${ }^{10}$ This relation is true only for continuous $t$ so that simulations with different $\tau$ values have to be performed in order to extrapolate to $\tau \rightarrow 0$ afterwards.

${ }^{11}$ This is indeed the only $c_{S W}$ contribution that enters into play at this order with trivial $W$ and $W^{\prime}$.
} 
with the analytical values in Table 1.

\begin{tabular}{|c|c|c|c|}
\hline$\theta$ & $c_{S W}^{(0)}=0.0$ & $c_{S W}^{(0)}=1.0$ & $c_{S W}^{(0)}=1.5$ \\
\hline 1.40 & $2.67621(4)$ & $1.67151(2)$ & $0.94999(1)$ \\
\hline 1.00 & $2.63837(3)$ & $1.64808(1)$ & $0.93229(1)$ \\
\hline 0.45 & $2.60727(3)$ & $1.62694(1)$ & $0.91948(1)$ \\
\hline 0.00 & 2.60571 & 1.62045 & 0.91067 \\
\hline
\end{tabular}

Table 1: Numerical results for $m_{q}^{(1)}$ on a $10^{3} * 21$ lattice with $c_{A}^{(0)}=c_{A}^{(1)}=0$ : the last line contains the infinite-volume results obtained from [7].

It is reassuring that, when varying $c_{S W}^{(0)}$, outputs change accordingly: the still-existing gap is explained by recalling that finite-size effects are still present and that the analytical results correspond to $m_{q}^{(0)}=0$ while in our simulations $m_{q}^{(0)} \neq 0$ due to the non-vanishing values of $\theta\left(m_{q}^{(0)}\right.$ approaches with decreasing $\theta^{12}$ the analytical infinite-volume values computed with $\theta=0.0$ ).

\section{Conclusions and acknowledgements}

According to the first, preliminary results, the outlined approach seems to be feasible: however, since different extrapolations (in $\tau$ and $L$ ) and interpolations (in $c_{A}^{(2)}$ and $c_{S W}^{(2)}$ when dealing with non-trivial $W$ and $W^{\prime}$ ) are needed, extra care will have to be paid not to spoil accuracy.

We warmly thank $L R Z$ centre (Munich) and $E C T^{*}$ (Trento) for providing us with computer time on their clusters.

This work was supported by the DFG SFB/TR 55.

\section{References}

[1] K. Symanzik, Continuum Limit and Improved Action in Lattice Theories. II. O(N) Nonlinear Sigma Model in Perturbation Theory, Nucl. Phys. B 226 (1983) 187.

[2] B. Sheikoleslami and R. Wohlert, Improved Continuum Limit Lattice Action for QCD with Wilson Fermions, Nucl. Phys. B 259 (1986) 572.

[3] R. Wohlert, Improved Continuum Limit Lattice Action For Quarks, unpublished.

[4] R. Horsley, H. Perlt, P.E.L. Rakow, G. Schierholz and A. Schiller, Perturbative determination of $c_{S W}$ for plaquette and Symanzik gauge action and stout link clover fermions, Phys. Rev. D 78 (2008) 054504. [hep-lat/0807.0345].

[5] M. Luscher, S. Sint, R. Sommer and P. Weisz, Chiral symmetry and $O(a)$ improvement in lattice QCD, Nucl. Phys. B 478 (1996) 365. [hep-lat/9605038].

[6] M. Luscher and P. Weisz, $O($ a) improvement of the axial current in lattice QCD to one loop order of perturbation theory, Nucl. Phys. B 479 (1996) 429. [hep-lat/ 9606016 ].

[7] H. Panagopoulos and Y. Proestos, The critical hopping parameter in $O(a)$ improved lattice QCD, Phys. Rev. D 65 (2002) 014511 [hep-lat/ 0108021 ].

[8] F. Di Renzo and L. Scorzato, Numerical Stochastic Perturbation Theory for full QCD, JHEP 0410 (2004) 073 [hep-lat/ 0410010$].$

\footnotetext{
${ }^{12}$ An analytical expression for $m_{q}^{(0)}$ can be found in section 3 of [6].
} 